\title{
Analysis of the Tuning Sensitivity of Silicon-on-Insulator Optical Ring Resonators
}

\author{
Tom Baehr-Jones, Michael Hochberg, Chris Walker, Eric Chan, Dennis Koshinz, William Krug, and Axel Scherer
}

\begin{abstract}
High-quality-factor optical ring resonators have recently been fabricated in thin silicon-on-insulator (SOI). Practical applications of such devices will require careful tuning of the precise location of the resonance peaks. In particular, one often wants to maximize the resonance shift due to the presence of an active component and minimize the resonance shift due to temperature changes. This paper presents a semianalytic formalism that allows the prediction of such resonance shifts from the waveguide geometry. This paper also presents the results of experiments that show the tuning behavior of several ring resonators and find that the proposed semianalytic formalism agrees with the observed behavior.
\end{abstract}

Index Terms-Integrated optics, integrated optoelectronics, optical waveguide, photothermal effects.

\section{INTRODUCTION}

$\mathbf{H}$ IGH-QUALITY-FACTOR ring resonators have recently been fabricated in thin silicon-on-insulator (SOI), with waveguide losses better than $7 \mathrm{~dB} / \mathrm{cm}$, and $Q$ values in excess of 45000 [1]. This suggests that these devices may find practical application as chemical sensors and as low-loss, very compact, add-drop optical multiplexers.

In general, any small change in the refractive index of the waveguide core or cladding material will lead to a shift in the resonance-peak locations. This refractive-index change might be an induced behavior, as might occur from applying a direct current (dc) electric field to an electrooptic material [2]. It might also simply be the result of a change in temperature of the entire device. Because of the narrowness of the high$Q$ peak widths, even a small shift in resonance-peak locations will completely change the effective behavior of the device. This is a blessing, in that even weakly active optical materials can provide switching capabilities. It is also a curse, in that a practical ring-based device will require careful temperature

Manuscript received August 23, 2004; revised May 13, 2005. This work was supported in part by the Defense Advanced Projects Research Administration contract N00421-02-D-3223 under the Chip-Scale Wavelength Division Multiplexing program.

T. Baehr-Jones, M. Hochberg, and C. Walker are with the California Institute of Technology, Pasadena, CA 91125 USA (e-mail: thorolf@ caltech.edu; hochberg@caltech.edu).

E. Chan, D. Koshinz, and W. Krug are with the Boeing Company, Renton, WA 98055 USA.

A. Scherer is with the Departments of Electrical Engineering, Applied Physics and Physics, California Institute of Technology, Pasadena, CA 91125 USA (e-mail: etcher@caltech.edu).

Digital Object Identifier 10.1109/JLT.2005.853147 control for consistent optical behavior. In fact, thermal-stability requirements are often the prime limitation on the practical application of optical ring resonators.

Since both the utility and limitations of optical ring resonators hinge on their tuning sensitivity, it is of exceeding importance to be able to predict this sensitivity based solely on the waveguide geometry. This paper presents a semianalytic formula that allows the prediction of the change in waveguide effective index based on changes in the refractive indices of arbitrary components of the waveguide. It is an unfortunate fact that the majority of high-index-contrast waveguide geometries do not have accurate closed-form expressions for either their dispersion diagrams or mode profiles. Thus, the proposed formula is based on the assumption that the mode profile and dispersion diagram is already known from a numerical model.

Two experiments have been performed to validate these predictions. First, the temperature dependence of the resonance peaks of ring resonators was observed. As one might expect, for even a few degrees Celsius, there was a large change in resonance. The proposed semianalytic formula matches the observed behavior quite well. Also observed was the change in resonance peaks due to the replacement of part of the waveguide cladding with an optical gel of known refractive index. Such a configuration matches well with what one might encounter in an active optically modulated system. Here, agreement between theory and experiment was still close, but not as good. The discrepancy is most likely due to the difficulty of sample preparation.

\section{Resonator Design And Predictions}

\section{A. Waveguide Geometry}

We will begin by presenting the waveguide geometry used. Our process starts with an SOI wafer with a $1.4 \mu \mathrm{m}$ thick buried oxide layer and a 120-nm silicon layer on top. We pattern our sample using electron-beam lithography. Our final waveguide is $0.5 \mu \mathrm{m}$ wide. Fig. 1 shows a diagram of the waveguide, as well as the modal pattern and the dispersion plot, obtained by solving the associated Hermetian eigenvalue problem [3].

We are able to obtain low-loss guiding with various choices of cladding. Even a completely unclad waveguide, with simply air above the buried oxide and the waveguide, will guide; a polymethylmethacrylate (PMMA) cladding will also work. In both cases, the waveguide loss is between 6 and $7 \mathrm{~dB} / \mathrm{cm}$ [1], of which about $4 \mathrm{~dB} / \mathrm{cm}$ can be attributed to leakage into 


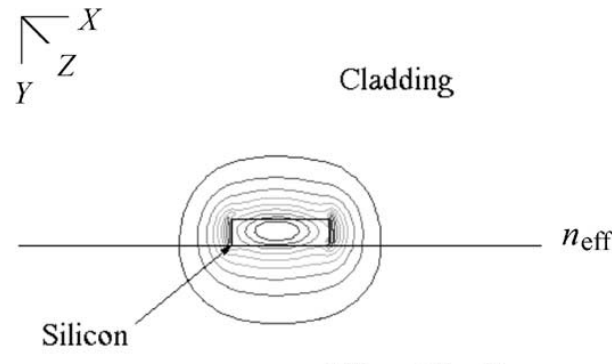

Silicon Dioxide

$0.5 \mu \mathrm{m}$

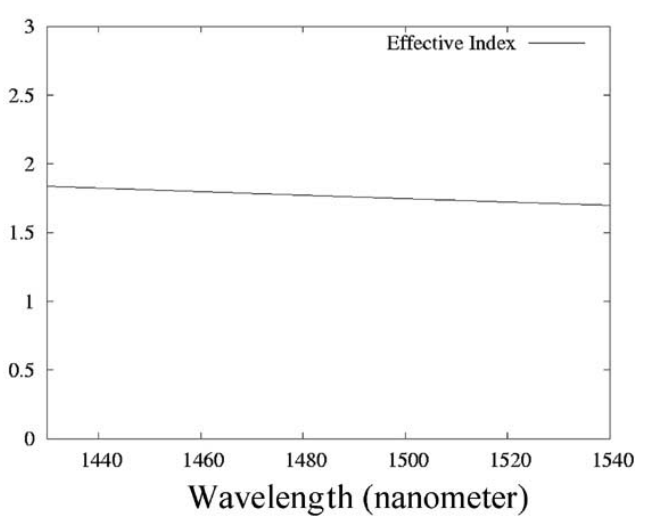

Wavelength (nanometer)

Fig. 1. Modal profile of the silicon waveguide and the dispersion plot. The profile is of $|E|$, with contour lines drawn in increments of $10 \%$ starting at $10 \%$ of the maximum field value. Part B shows effective index as a function of wavelength.
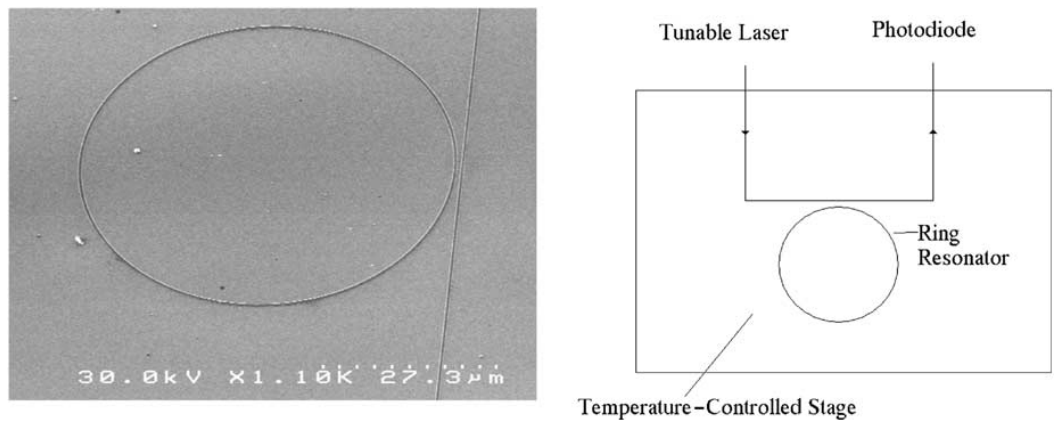

Fig. 2. Scanning electron micrograph of one of the ring resonators studied and a logical diagram of the experimental setup.

the silicon handle due to perturbation theory. Only one wellcontained optical mode is supported in the 1.4-1.6 $\mu \mathrm{m}$ spectral range. This mode has the electric field polarized primarily in the horizontal direction.

It is possible to predict the exact effect that an index change in a given region will have on the effective index of the mode. For clarity, we will term the value $\partial n_{\mathrm{eff}} / \partial \varepsilon_{\mathrm{c}}$ the effective index susceptibility, a dimensionless parameter, which represents the change in total modal refractive index for a differential amount of uniform change in the relative dielectric constant of some part of the waveguide geometry. We present the result here, with the complete derivation via perturbation theory presented in Appendix I.

$$
\frac{\partial n_{\mathrm{eff}}}{\partial \varepsilon}=\frac{\iint|E|^{2} \mathrm{~d} x \mathrm{~d} y}{2 \sqrt{\frac{\mu_{0}}{\varepsilon_{0}}} \iint \operatorname{Re}\left(E^{*} \times H\right) \cdot e_{z} \mathrm{~d} x \mathrm{~d} y} .
$$

Equation (1) presents a convenient analytic method to predict the shift in the modal effective index induced by a small change in the refractive index in some part of the mode. The integral in the numerator of (1) is taken over the region where the refractive index is changing, while the integral in the denominator is taken over the entire $x-y$ plane. This is done assuming, of course, that propagation occurs in the $z$ direction. The International System of Units (SI) unit system is used.

As an example, for the thermal tuning results we present, this integral would be taken over the core silicon strip only. This is because the changes in index in the Silicon Dioxide and the cladding as a function of temperature can be ignored as compared to that of Silicon. In cases where there are multiple components of the waveguide with different relative changes in the indices of refraction, such as might be the case for a material with a temperature gradient, (1) can still be applied. It should be broken into multiple regions, producing different susceptibilities for different regions.

Note that the application of (1) requires that the exact modal pattern be known. Fortunately, this is known via our eigensolver. As a result, we are in the position to utilize (1) to predict the exact change in effective index that a refractive-index change in a waveguide component might have.

\section{B. Resonator Design and Measurement}

The waveguide geometry in Fig. 1 has bend losses that become negligible with a bend radius greater than about $30 \mu \mathrm{m}$. We fabricate a resonator by constructing a circle with these waveguides, with a bend radius of $30 \mu \mathrm{m}$. Evanescent coupling from a straight waveguide can be used to couple into and out of these resonators. Fig. 2 details the layout of such devices and shows a scanning electron micrograph (SEM) of a typical device.

Also shown in Fig. 2 is the experimental setup used to measure the devices. As can be seen, a computer-controlled tunable laser, along with a photodiode, is used to interrogate the wavelength-dependent transmission spectrum of the resonator. Our input couplers are fairly broadband and have an approximate transmission uncertainty of $3 \mathrm{~dB}$. Since our results are all based on a very fine spectral feature, however, they are more or less independent of the particular spectral dependence of the 
efficiency of the input coupler. For the temperature-dependent measurements, the stage on which the entire sample rested was temperature controlled.

\section{Ring Resonator Theory}

In order to interpret the measurements we will show, it is important to understand the behavior of the ring resonators in the presence of varying refractive indices. We base our analysis on the formalism presented in [4], but also account for waveguide dispersion. A resonance peak will always obey the following relationship:

$$
\frac{L}{m}=\frac{\lambda}{n_{\mathrm{eff}}(\lambda)} .
$$

Here, $L$ is the effective path length around the resonator, $m$ is an integer indicating the order of the resonance, $\lambda$ is the free-space wavelength of the resonance frequency, and $n_{\text {eff }}(\lambda)$ is the effective index of the waveguide mode involved in the resonance. Note that it is essential to allow $n$ to vary with the wavelength to obtain an accurate model. A given resonance peak will have a constant $m$, while $\lambda$ and $n_{\text {eff }}(\lambda)$ will vary with changes in the waveguide refractive index.

We must consider the behavior of $n_{\text {eff }}(\lambda)$ in the presence of both a varying temperature and wavelength. The total differential of $n_{\mathrm{eff}}$ is given as

$$
\mathrm{d} n_{\text {eff }}=\left(\frac{\partial n_{\text {eff }}}{\partial \varepsilon_{\mathrm{c}}}\right)\left(\frac{\partial \varepsilon_{\mathrm{c}}}{\partial T}\right) \mathrm{d} T+\left(\frac{\partial n_{\mathrm{eff}}}{\partial \lambda}\right) \mathrm{d} \lambda .
$$

Here, we take $\varepsilon_{\mathrm{c}}$ as the relative dielectric constant for the material that is changing with temperature. In this case we assume that the silicon waveguide core's temperature dependence is well known, as mentioned before. The other differential involved with the $\mathrm{d} T$ term is, of course, the result of (1) for our waveguide mode. Note that we have also allowed for wavelength dispersion. Equation (3) can be combined with (2) to yield the resonance wavelength as a function of temperature.

$$
\begin{aligned}
\lambda=\frac{L}{m}\left(1-\left(\frac{L}{m}\right)\left(\frac{\partial n_{\mathrm{eff}}}{\partial \lambda}\right)\right)^{-1}\left(\frac{\partial n_{\mathrm{eff}}}{\partial \varepsilon_{\mathrm{c}}}\right) \\
\quad \times 2 n_{\mathrm{si}}\left(\frac{\partial n_{\mathrm{si}}}{\partial T}\right)\left(T-T_{0}\right)+\frac{L}{m} n_{\mathrm{eff}}\left(\lambda_{0}\right) .
\end{aligned}
$$

Here, we take $T_{0}$ to be the lowest temperature that the ring was measured at, which in our case was $20^{\circ} \mathrm{C}$; then, $\lambda_{0}, m$, and $n_{\text {eff }}\left(\lambda_{0}\right)$ are the resonance peak under study observed at this temperature. This expression suggests that when plotted as a function of temperature, the position of a given resonance peak should be linear. The slope is dependent on the product of wellknown physical constants and the results of our modesolver and perturbation theory; in particular, the temperature derivative of the index of refraction of silicon is known to be $1.5 \times 10^{-4} 1 / \mathrm{K}$ at room temperature [5]. We did not use a cladding on the ring resonators for the temperature tuning; that is, above the waveguide there was simply air, and below the waveguide there
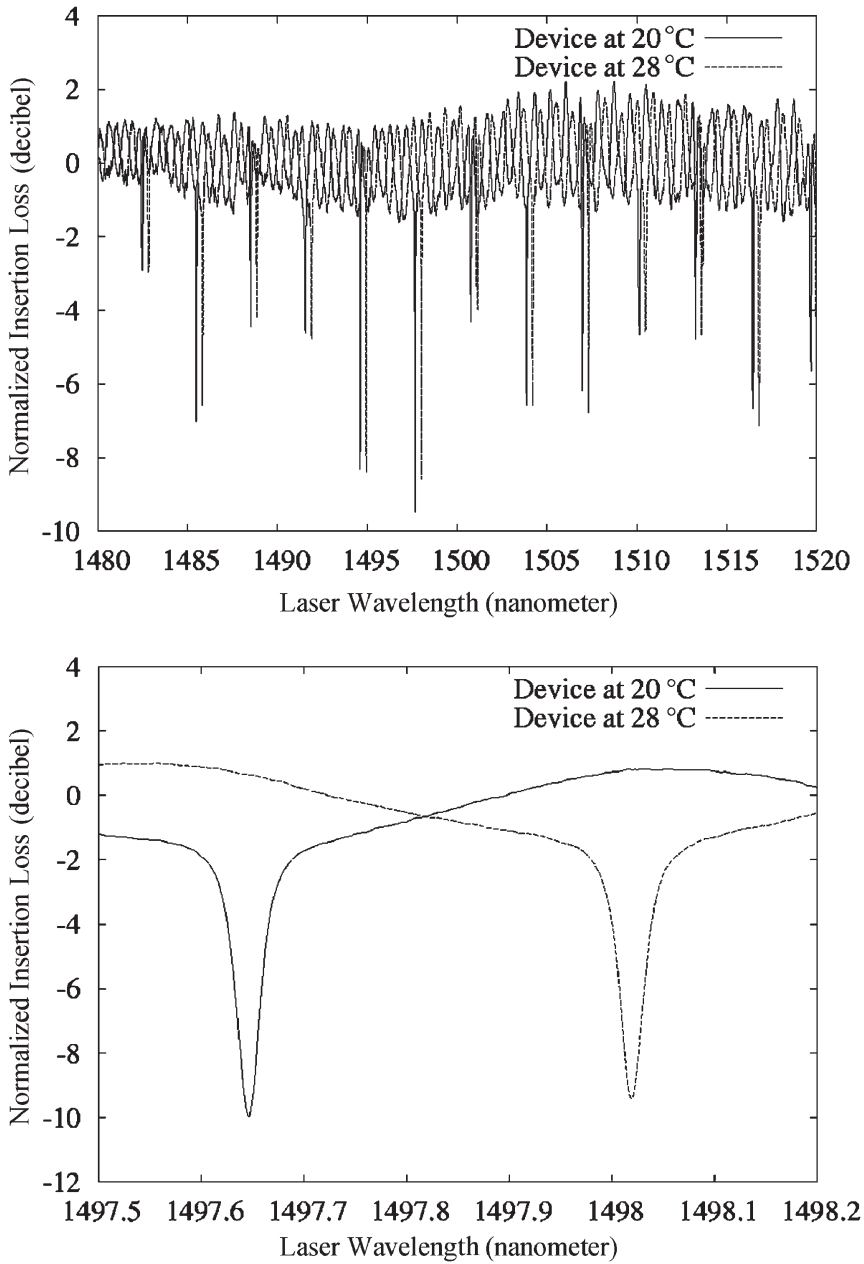

Fig. 3. Normalized fiber-to-fiber transmission in decibels as a function of wavelength in nanometers for two different temperatures for one of the ring resonators studied. The transmission has been normalized against our baseline fiber-to-fiber coupling efficiency. A detailed plot of the peaks near $1498 \mathrm{~nm}$ is shown.

was silica. Since the silica has a temperature derivative of the index one order of magnitude less [6] than silicon, it is ignored for the purposes of expression (4).

For the index-matching measurements, (4) must be rewritten slightly, as follows:

$$
\begin{aligned}
\lambda=\frac{L}{m}\left(1-\left(\frac{L}{m}\right)\left(\frac{\partial n_{\mathrm{eff}}}{\partial \lambda}\right)\right)^{-1} & \\
& \times\left(\frac{\partial n_{\mathrm{eff}}}{\partial \varepsilon_{\mathrm{c}}}\right) \Delta \varepsilon_{\mathrm{c}}+\frac{L}{m} n_{\mathrm{eff}}\left(\lambda_{0}\right) .
\end{aligned}
$$

Now, the independent variable is simply the shift in relative dielectric constant due to the change in the index-matching gel. Significantly, the susceptibility term $\partial n_{\mathrm{eff}} / \partial \varepsilon_{\mathrm{c}}$ is now the susceptibility in cladding index shift. In this case, the domain of integration in (1) consisted of the entire cladding region, and not including the core Silicon strip or the Silicon Dioxide layer. Matching (4) and (5) to two different experiments thus provides an excellent validation of the perturbative approach.

Let us list the particular values of $\partial n_{\text {eff }} / \partial \varepsilon_{\mathrm{c}}$ for our waveguide geometry for the situations we consider, near $1.55 \mu \mathrm{m}$. Using the results of a numerical eigensolver, the integral in 


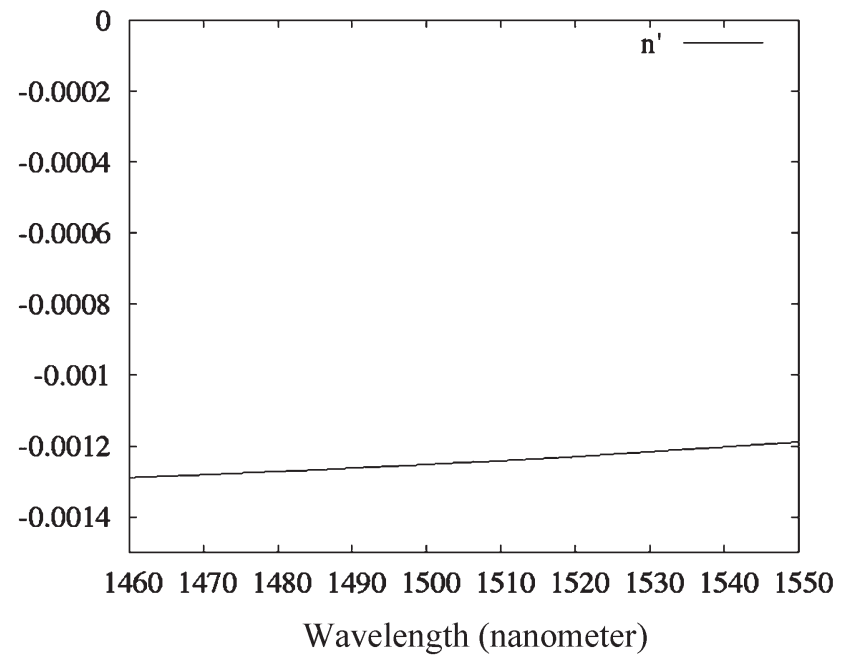

(a)

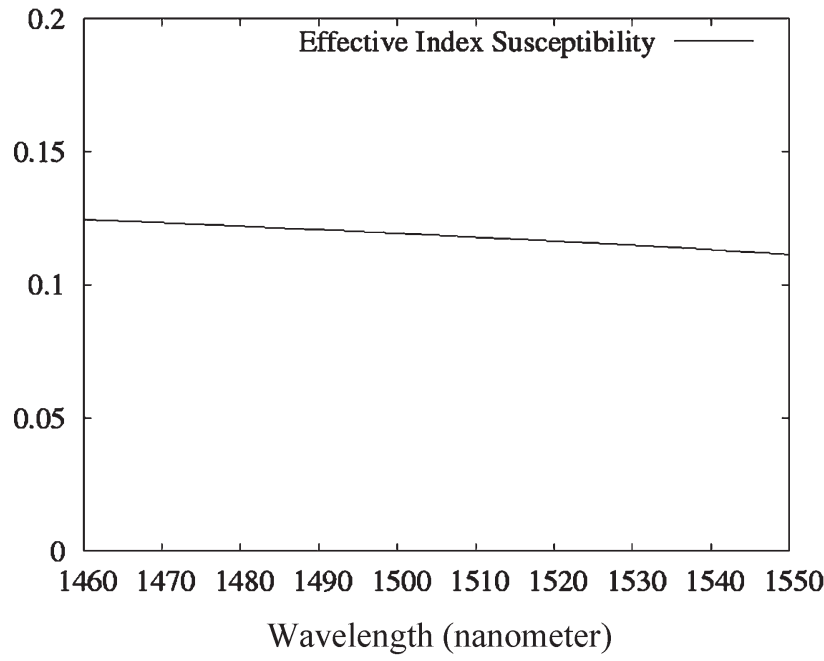

(b)

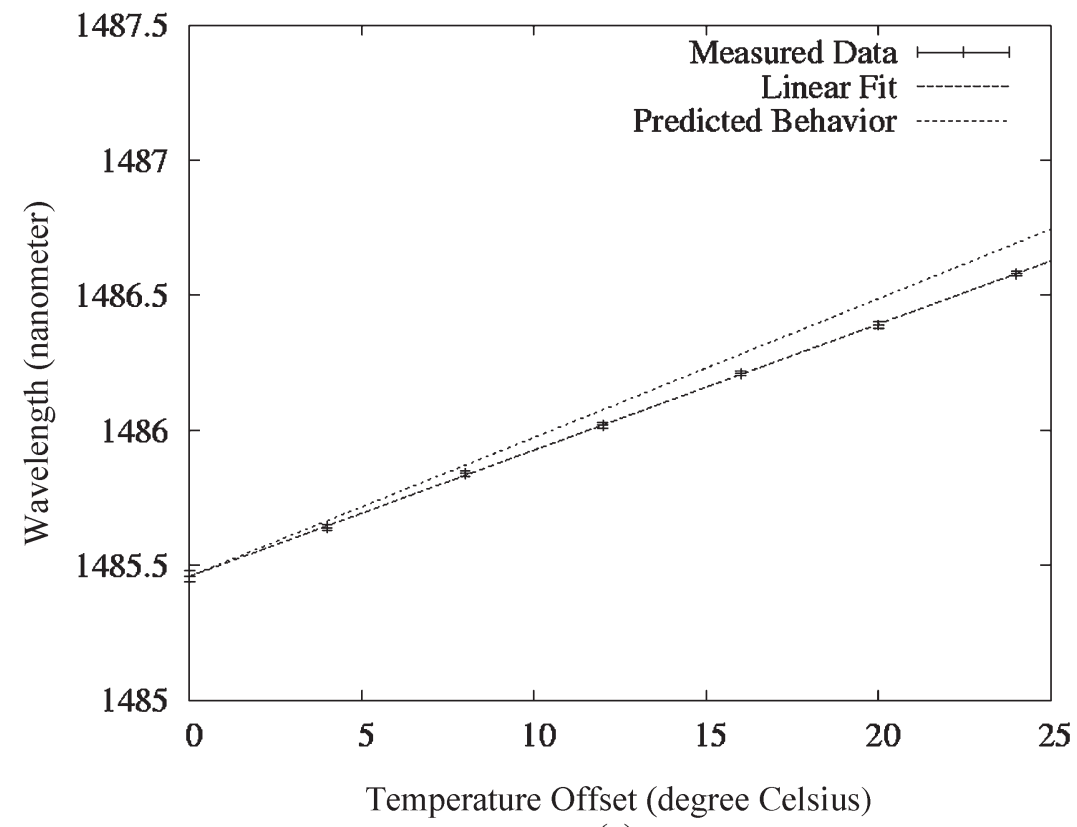

(c)

Fig. 4. Panel (a) shows $\mathrm{d} n / \mathrm{d} \lambda$ versus wavelength in $1 / \mathrm{nm}$, while (b) list the simulated susceptibility to core changes, a dimensionless quantity as a function of wavelength. In (c), we show the movement of a resonance peak as a function of temperature offset, in degrees Celsius. The base temperature was $20{ }^{\circ} \mathrm{C}$. Also shown is the predicted behavior.

(1) was applied, for the temperature susceptibility, just to the silicon strip. The result was 0.11 , a dimensionless quantity. For the index-matching susceptibility, the integral in (1) is taken over just the cladding region, not including the silicon strip or the substrate, yielding 0.13 .

\section{RESUlts}

The dependence of the location of the resonance peaks on temperature and cladding index was measured to verify our semianalytic method. In both cases, the experimental procedure was straightforward; the transmission spectra were measured as a function of varying temperature or index-matching gel. The location of a resonance peak was traced and then the results plotted with the resonance shift as a function of cladding index or temperature. As can be seen from (4) and (5), there should be a linear dependence observed.
The temperature-tuning experiments involved, as previously mentioned, controlling the temperature of the stage upon which the sample rested. We estimate the uncertainty of our temperature control to be about $0.1^{\circ} \mathrm{C}$. A typical set of transmission spectra that were observed can be seen in Fig. 3. All of the spectra that we show are normalized to remove the unimportant insertion loss spectrum of our measurement setup.

We repeated such measurements several times for each temperature value on one device, to allow the estimation of the characteristic measurement uncertainty that our system had. We measured the temperature dependence of a second ring resonator as well, but taking only one data point for each temperature. Both devices consisted of $30 \mu \mathrm{m}$ bend-radius ring resonators. The ring-to-input-waveguide spacing was $0.37 \mu \mathrm{m}$, edge to edge. The $Q$ in this case was approximately 20000 . As previously mentioned, these rings were air clad. During our 
(a)

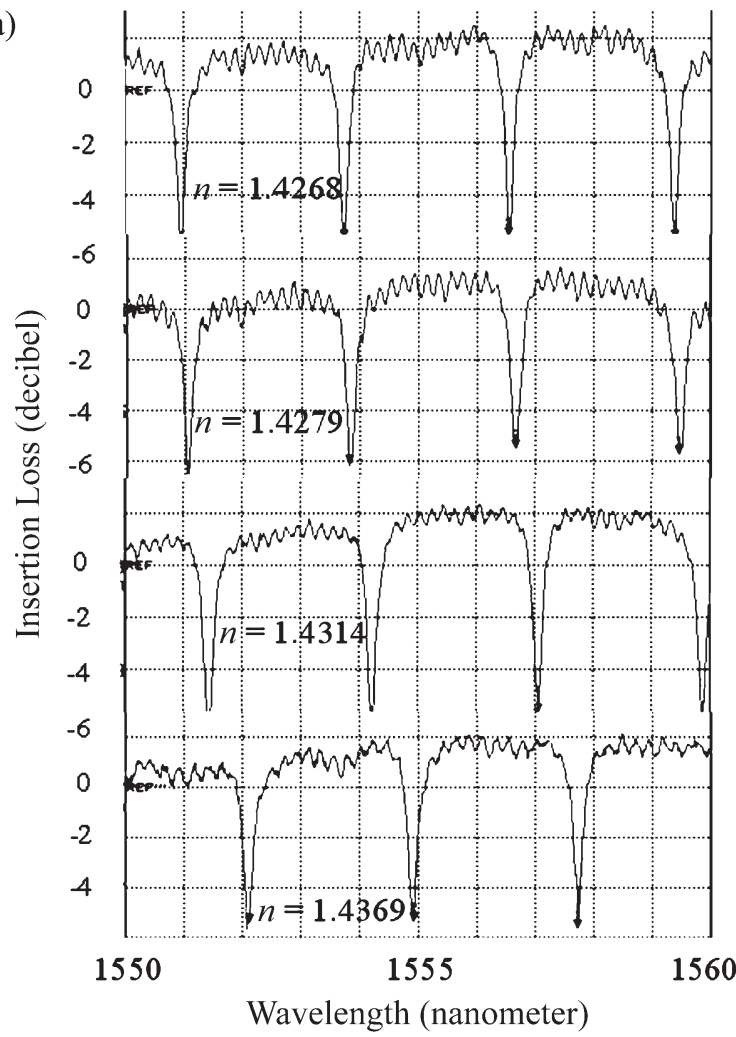

(b)

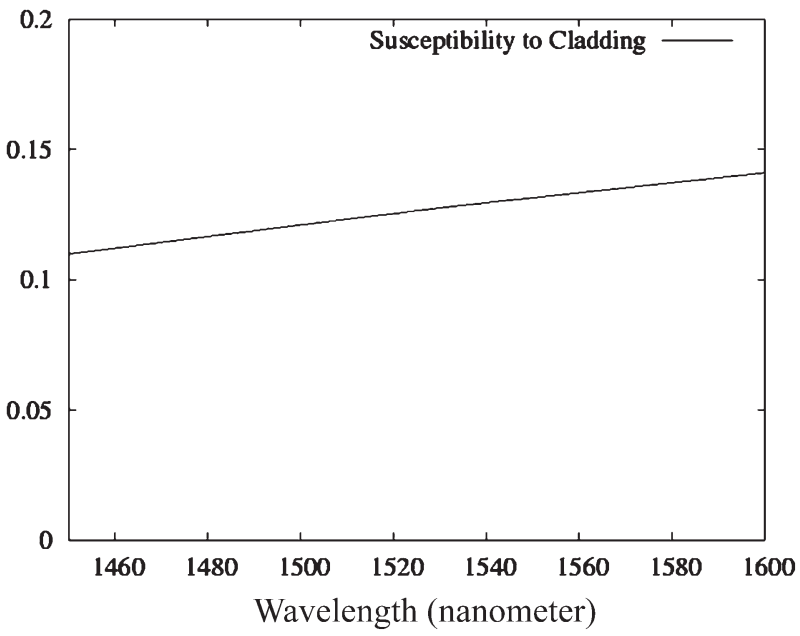

(c)

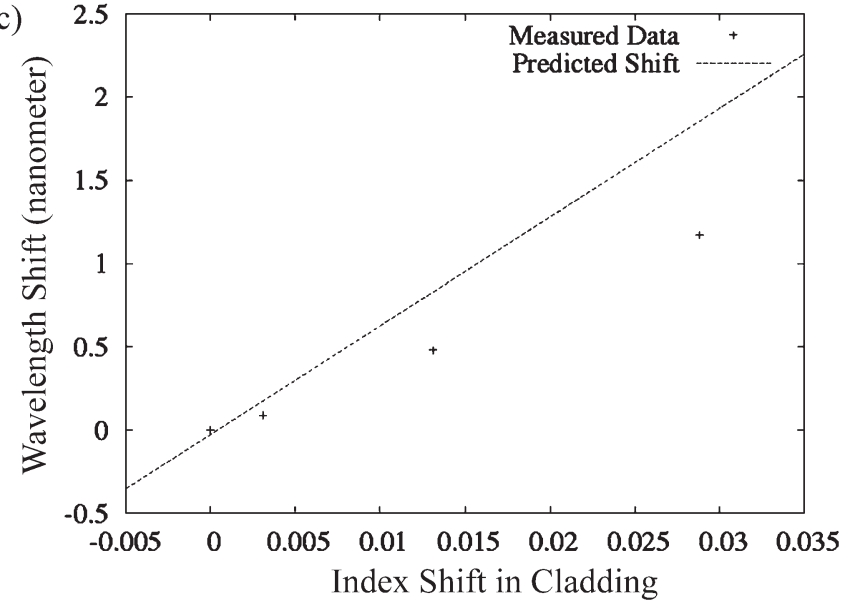

Fig. 5. Panel (a) shows a series of optical scans for various cladding indices. Panel (b) shows the effective index susceptibility to changes in cladding as a function of wavelength, while (c) shows the expected and observed shifts in resonance peaks for several different index matching gels.

experiments, meaningful changes in the $Q$ 's and the extinctions of the resonance peaks were not observed to the limits of our testing apparatus.

We will summarize the results of both the temperature-tuning and also the index-matching experiments at the end of this section for easy comparison. Here in Fig. 4, we show plots of the various quantities involved in the prediction of the temperature tuning and then the measured data along with the predicted linear dependence.

There is a small discrepancy between the results and the predicted behavior, which cannot be attributed to the uncertainty of measurement. This systemic error is most likely due to a combination of a slight simulation error, and possibly an incorrect value used for the base refractive index of silicon; the value 3.46 was used in our simulations. In any case, the results do match fairly closely, indicating that our modesolver accurately predicts the modal pattern and that (1) correctly predicts the effect of refractive-index change on the effective index.

The index-matching gel experiments consisted of measuring the transmission spectra of a ring device with a gel of known index deposited on the sample. Then, the sample was cleaned and the gel removed, and a material with another index was deposited, and the sample remeasured. Because of the difficulty
TABLE I

Summary of TUNing MEASUREMENTS AND PREDICTIONS

\begin{tabular}{|c|l|c|c|c|}
\hline Device & $\begin{array}{c}\Lambda_{O} \\
\text { (nanometer) }\end{array}$ & $\begin{array}{c}M \\
\text { (nanometer per } \\
\text { degree Kelvin) } \\
\text { Theory }\end{array}$ & $\begin{array}{c}M \\
\text { (nanometer per } \\
\text { degree Kelvin) } \\
\text { Measured }\end{array}$ & $\chi^{2}$ Reduced \\
\hline 1 & $1485.46 \pm 0.008$ & 0.0514 & $0.0467 \pm 0.0005$ & 0.203 \\
\hline 1 & $1503.85 \pm 0.009$ & 0.0512 & $0.0463 \pm 0.0006$ & 0.059 \\
\hline 1 & $1529.36 \pm 0.009$ & 0.0502 & $0.0463 \pm 0.0005$ & 0.153 \\
\hline 2 & 1506.91 & 0.0512 & 0.0472 & \\
\hline 3 & 1553.7 & $65.3(\mathrm{~nm})$ & $41.01(\mathrm{~nm})$ & \\
\hline
\end{tabular}

Devices 1 and 2 show results of temperature sweeps for several resonance peaks from two ring resonator devices. $M$ is taken to be the slope of the line giving the movement of a resonance peak is a function of temperature. Device 3 was subjected instead to a shift in cladding index, and has a slope with the dimension in nanometers, as the shift in relative dielectric constant is dimensionless.

of sample preparation, far fewer data points were taken. In Fig. 5, we present a series of spectra that were taken for various index-matching gels, as well as the measured peak deflection plotted against the theoretical prediction.

Agreement between theory and data is not as good here as for thermal tuning. The source of this discrepancy is unknown, but the authors speculate that it is related to the repeated coating and decoating of the samples in these experiments. The results of both the temperature tuning and index matching experiments are presented in Table I. 


\section{CONCLUSION}

The authors have developed and demonstrated experimentally a semianalytic formalism for predicting the tuning sensitivity of optical ring microresonators. The proposed formalism can be used to predict how much modulation can be achieved from a modification in the index of an active component in the waveguide. It can also be used to predict how much unwanted resonance shift will occur due to a change in resonator temperature. It is hoped that the proposed approach will prove valuable to those performing similar work.

\section{APPENDIX I \\ DEVELOPMENT OF EFFECTIVE INDEX PERTURBATION EXPRESSION}

Here we show the complete derivation of formula (1). We start our approach by assuming a time dependence of $\exp (-i w t)$ and a continuous $z$ symmetry in the structure under consideration, with a field dependence of $\exp (i \beta z)$. Maxwell's equations (in SI units) can then be recast into a generalized eigenvalue equation of the form

$$
H \Psi=i \beta A \Psi
$$

where $\Psi$ is a six-vector representing the $E$ and $H$ fields in the $x-y$ plane, and the two tensor operators $H$ and $A$ have the forms shown in (7) at the bottom of the page.

Let us make some basic observations about these operators: $H$ is anti-Hermetian, as long as $\varepsilon(x, y)$ is real. $A$ is Hermetian, but not positive definite and clearly singular. Basic linear al- gebra dictates that if $\varphi_{0}$ and $\varphi_{1}$ are generalized eigenvectors of $H$ with different eigenvalues, they will be orthogonal in the $A$ norm. Note that the $A$ norm, of course, is not a proper norm, since it has negative and zero eigenvalues. Considering the field dependence assumed, it is clear that an eigenvalue of $H$ with a positive imaginary eigenvalue is a forward-propagating mode, while a negative imaginary eigenvalue is a negativepropagating mode. The effective index of such modes is given as $|\beta /(w / c)|$. All eigenvalues must be purely imaginary due to $H$ being anti-Hermetian. One can note that the $A$ norm corresponds to the amount of power being propagated in a mode, to a constant factor. Finally, we note the useful relation by which an eigenvalue can be extracted from the eigenvector

$$
\beta=\frac{1}{i} \frac{\Psi^{t} H \Psi}{\Psi^{t} A \Psi} .
$$

Let us begin by assuming that (7) has been solved for a given $\Psi$ and $\beta$. Let us further assume that $\Psi$ is the only eigenvector of $H$ that has $\beta$ as an eigenvalue. This is, in practice, an easily achievable condition, since for most real problems $H$ is almost never degenerate in the region of interest. Even if it is, all the degenerate modes can be solved for and orthogonalized, as long as the effective index implied by $\beta$ exceeds that of all cladding indices.

One of the attractions of this formalism is that the $H$ operator is linear in changes in relative dielectric constants. As a result, we can represent the effect of a perturbation in relative dielectric constant $\Delta \varepsilon(x, y)$ as that shown in (9) at the bottom of the page.

$$
\begin{aligned}
& H=\left(\begin{array}{cccccc}
i w \varepsilon_{0} \varepsilon(x, y) & 0 & 0 & 0 & 0 & \partial_{y} \\
0 & i w \varepsilon_{0} \varepsilon(x, y) & 0 & 0 & 0 & -\partial_{x} \\
0 & 0 & i w \varepsilon_{0} \varepsilon(x, y) & -\partial_{y} & \partial_{x} & 0 \\
0 & 0 & -\partial_{y} & i w \mu_{0} & 0 & 0 \\
0 & 0 & \partial_{x} & 0 & i w \mu_{0} & 0 \\
\partial_{y} & -\partial_{x} & 0 & 0 & 0 & i w \mu_{0}
\end{array}\right) \\
& A=\left(\begin{array}{cccccc}
0 & 0 & 0 & 0 & 1 & 0 \\
0 & 0 & 0 & -1 & 0 & 0 \\
0 & 0 & 0 & 0 & 0 & 0 \\
0 & -1 & 0 & 0 & 0 & 0 \\
1 & 0 & 0 & 0 & 0 & 0 \\
0 & 0 & 0 & 0 & 0 & 0
\end{array}\right) .
\end{aligned}
$$

$$
\begin{aligned}
H^{\prime} & =H+\Delta H \\
\Delta H & =\left(\begin{array}{cccccc}
i w \varepsilon_{0} \Delta \varepsilon(x, y) & 0 & 0 & 0 & 0 & 0 \\
0 & i w \varepsilon_{0} \Delta \varepsilon(x, y) & 0 & 0 & 0 & 0 \\
0 & 0 & i w \varepsilon_{0} \Delta \varepsilon(x, y) & 0 & 0 & 0 \\
0 & 0 & 0 & 0 & 0 & 0 \\
0 & 0 & 0 & 0 & 0 & 0 \\
0 & 0 & 0 & 0 & 0 & 0
\end{array}\right) .
\end{aligned}
$$


If we could solve the eigenvalue problem for $H^{\prime}$, then we would know the precise impact of a change in relative index on the effective index. Let us assume that such a solution has been found, and due to the presumed smallness of $\Delta \varepsilon(x, y)$, it can be written as

$$
(H+\Delta H)(\Psi+\Delta \Psi)=i(\beta+\Delta \beta) A(\Psi+\Delta \Psi)
$$

where all terms that involve $\Delta$ are small. We can express the new eigenvalue using (8) as follows:

$$
\beta+\Delta \beta=\frac{1}{i} \frac{(\Psi+\Delta \Psi)^{t}(H+\Delta H)(\Psi+\Delta \Psi)}{(\Psi+\Delta \Psi)^{t} A(\Psi+\Delta \Psi)} .
$$

However, we must have $\Delta \Psi$ be orthogonal to $\Psi$, except possibly to the extent that $\Delta \Psi$ contains a component directly along $\Psi$, which does not affect the outcome. Using this fact, and expanding (11) to first order and using (6) and the antiHermetian property of $H$, we obtain

$$
\Delta \beta=\frac{1}{i} \frac{\Psi^{t} \Delta H \Psi}{\Psi^{t} A \Psi} .
$$

If we assume that $\Delta \varepsilon$ is nonzero only in a given region and takes on a uniform value here, (12) expands into (1).

\section{REFERENCES}

[1] T. Baehr-Jones, M. Hochberg, C. Walker, and A. Scherer, "High-Q resonators in thin Silicon-on-Insulator," Appl. Phys. Lett., vol. 85, no. 16, pp. 3346-3347, Oct. 2004.

[2] L. R. Dalton, B. H. Robinson, A. K. Y. Jen, W. H. Steier, and R. Nielsen, "Systematic development of high bandwidth, low drive voltage organic electro-optic devices and their applications," Opt. Mater, vol. 21, pp. 19-28, Jan. 2003.

[3] J. D. Joannopoulos, R. D. Meade, and J. N. Winn, Photonic Crystals. Princeton, NJ: Princeton Univ. Press, 1995.

[4] D. Rafizadeh, J. P. Zhang, R. C. Tiberio, and S. T. Ho, "Propagation loss measurements in semiconductor microcavity ring and disk resonators," J. Lightw. Technol., vol. 16, no. 7, pp. 1308-1314, Jul. 1998.

[5] A. Wolfe and G. Zissis, The Infrared Handbook. Ann Arbor, MI: Environmental Research Institute of Michigan, 1989.

[6] R. Sosran, Properties of Silica. New York: American Chemical Soc., 1927, Monograph Series.

Tom Baehr-Jones was born in New York, NY, in 1980. He received the B.S. degree in physics from the California Institute of Technology, Pasadena, in 2002. He is currently a doctoral candidate at the California Institute of Technology.

He cofounded Simulant, Inc., in 2000, where he developed finite-difference time-domain (FDTD)-based simulation software, particularly aimed at helping design integrated optical components. He cofounded Luxtera, Inc., in 2001, a firm that specializes in the design of integrated optics components. There, he helped design such components with the assistance of FDTD software. His current research interests include the study of integrated optical devices, and efficient methods to simulate Maxwell's equations in various circumstances.
Michael Hochberg was born in Ithaca, NY, in 1980. He received the B.S. degree in physics from the California Institute of Technology, Pasadena, in 2002. $\mathrm{He}$ is currently a doctoral candidate at the California Institute of Technology.

He cofounded Simulant, Inc., in 2000, and acted as CEO until its acquisition. He was a Cofounder at Luxtera, Inc., a firm that specializes in the design of integrated optics components. There, he assisted with the prototyping of various photonic devices. He is working on the synthesis and fabrication of novel nanooptical devices.

Chris Walker, photograph and biography not available at the time of publication.

Eric Chan received the B.S. degree in E.E.C.S. from the University of California, Berkeley, in 1973, and the M.S. and Ph.D. degrees in E.E. from Columbia University, New York, in 1975 and 1980, respectively.

From 1979 to 1987, he was a Senior Member of Technical Staff of the Optoelectronic Group at the AT\&T Engineering Research Center, Princeton, NJ. He joined Boeing in 1987. Currently, he is a Boeing Technical Fellow working with the Optical Technology Group of Boeing Phantom Works. His research interests include design, packaging, and characterization of optoelectronic devices for aerospace applications.

Dennis Koshinz, photograph and biography not available at the time of publication.

William Krug received the Ph.D. degree in applied physics and organic chemistry from the Johns Hopkins University, Baltimore, MD, in 1978.

He joined the Boeing High Tech Center of Gould, Inc., in 1986 to lead research in nonlinear optical switching. He is an Associate Technical Fellow for Optical Technology with Boeing Phantom Works. He is a PI for a Chip-Scale Wavelength Division Multiplexing (WDM) DARPA program, focusing on tunable silicon-on-insulator (SOI) optical devices for scalable rapidly configurable wavelength routing system-on-chips for mobile platforms. He previously demonstrated thin- and thick-film sonar and chemical sensors, batteries, and switches.

Axel Scherer received the B.S., M.S., and Ph.D. degrees from the New Mexico Institute of Mining and Technology, Socorro, in 1981, 1982, and 1985 , respectively.

From 1985 until 1993, he worked in the Quantum Device Fabrication Group at Bellcore. Currently, he is the Bernard E. Neches Professor of Electrical Engineering, Applied Physics, and Physics at the California Institute of Technology, Pasadena, specializing in device microfabrication. His research interests include design and fabrication of functional photonic, nanomagnetic, and microfluidic devices. 